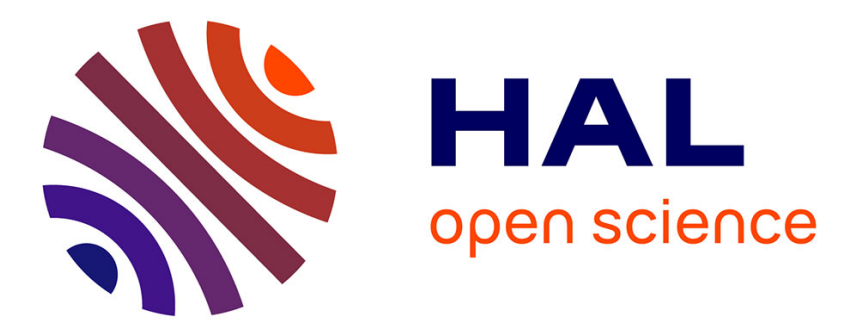

\title{
Towards model-based approaches for musical instruments making: validation of the model of a Spanish guitar soundboard and characterization features proposal
}

Romain Viala, Marco A Pérez, Vincent Placet, Antonio Manjon, Emmanuel Foltête, Scott Cogan

\section{To cite this version:}

Romain Viala, Marco A Pérez, Vincent Placet, Antonio Manjon, Emmanuel Foltête, et al.. Towards model-based approaches for musical instruments making: validation of the model of a Spanish guitar soundboard and characterization features proposal. Applied Acoustics, 2021, 172, pp.107591. 10.1016/j.apacoust.2020.107591 . hal-03186581

\section{HAL Id: hal-03186581 https://hal.science/hal-03186581}

Submitted on 31 Mar 2021

HAL is a multi-disciplinary open access archive for the deposit and dissemination of scientific research documents, whether they are published or not. The documents may come from teaching and research institutions in France or abroad, or from public or private research centers.
L'archive ouverte pluridisciplinaire HAL, est destinée au dépôt et à la diffusion de documents scientifiques de niveau recherche, publiés ou non, émanant des établissements d'enseignement et de recherche français ou étrangers, des laboratoires publics ou privés. 


\title{
Towards model-based approaches for musical instruments making : validation of the model of a Spanish guitar soundboard and characterization features proposal
}

\author{
Romain Viala ${ }^{\mathrm{a}}$, Marco A. Pérez ${ }^{\mathrm{b}}$, Vincent Placet ${ }^{\mathrm{a}}$, Antonio Manjón ${ }^{\mathrm{c}}$, \\ Emmanuel Foltête ${ }^{a}$, Scott Cogan ${ }^{a}$ \\ ${ }^{a}$ Univ. Bourgogne Franche-Comté, FEMTO-ST Institute, CNRS/UFC/ENSMM/UTBM \\ Department of Applied Mechanics, 25000 BESANÇON-FR, Tel. : +(33 3)81666010 \\ ${ }^{b} I Q S$ School of Engineering, Universitat Ramon Llull, Via Augusta 390, 08017 Barcelona, \\ Spain \\ ${ }^{c}$ Guitar-Maker, Av. de la Playa 84, Sant Adrià del Besós, 08930 Barcelona, Spain
}

\begin{abstract}
Nowadays, the virtual prototyping method is widely used for industrial applications and can lead to a powerful tool for musical instruments making and conservation. Nevertheless, physics-based models of musical instruments are barely developed for this purpose and the confrontation between model predictions and experiments have been the focus of very few researches. The objective of this paper is to highlight the predictive capability of physics-based models in dynamic domain, even in presence of variable by nature material and climatic conditions. For this purpose, a finite element model of the soundboard of a Spanish guitar is developed for model validation purposes. The simulated modal bases are compared with experimental ones from a previous study. Screening and stochastic analyses are performed to rank which are, among material and climatic parameters, the most influential ones on the dynamics of guitar soundboard. Moreover, uncertainties are taken into account to evaluate the dispersion of the response for a given design, and simulations are validated facing experimental data. It is shown that specific elastic parameters of the wood (in longitudinal and radial directions and longitudinal-radial plane) of the top plate are mainly
\end{abstract}

Email address: romain.viala@univ-fcomte.fr () 
influential with regard to the dynamics of the soundboard, and the relative humidity changes have a non negligible impact. Moreover, test-model correlations have shown that a nominal model with average material parameters is able to predict the dynamical behaviour of a real braced soundboard with an average error on the first eight eigenfrequencies lower than $4 \%$. In addition, when uncertainties are taken into account, the model is able to predict every experimental data. Finally, dynamic features like CFDAC and Fuzzy-FRFs are proposed in an innovative way in this application domain.

Keywords: Model validation, Uncertainty quantification, Modal analysis, Screening analysis, Musical acoustics, Guitar soundboard

\section{Higlights}

- Model validation of musical instrument part even in presence of strong uncertainties

— Fuzzy-FRF method illustrated to musical acoustics domain

- Screening analysis of influential material and climatic parameters on guitar soundboard dynamics for fixed geometry

- Similar impact of $\frac{E_{L}}{\rho}, \frac{E_{R}}{\rho}, \frac{G_{L R}}{\rho}$ and relative humidity on eigenfrequencies of guitar soundboard in free conditions

- Predominance of $\frac{E_{L}}{\rho}$, followed by $\frac{E_{R}}{\rho}, \frac{G_{L R}}{\rho}$ on eigenmode shapes

\section{1. Introduction}

The guitar, electric or acoustic, is one of the most popular musical instrument all over the world. It has been used in almost every musical style since the $X X^{t h}$ century and is often the first choice for musical learning. The guitar clusters a wide range of prices and qualities. One main family is considered here, the acoustic guitars. The acoustic guitars transfer the vibratory energy of the strings partly into an acoustic energy through their sounding box and the radiation of their flat parts, especially soundboards. 
It is usually considered that the soundboard affects mainly the acoustic behaviour of the guitars [1], [2]. The shape and thickness of the soundboard, as well as the characteristics of the braces (number, orientation, shapes) affect the mechanical behaviour of the guitar in both the static and dynamic domain. In the static domain, the top plate has to sustain the strings tension, and the mechanical characteristics of both plate and braces affect the stiffness of the system. In the dynamical domain, the eigenmodes of the soundboard are also strongly linked with the mechanical behaviour of the parts. These different design and material choices, that depend on the instrument maker, impact the resonance modes and acoustical features of the instrument. However, these parameters are barely quantified and studied from an objective point of view, since the construction process is based mainly on tradition and empiricism.

Generally, mechanically based works study acoustic guitars by experimental, analytical and numerical means, mainly focusing on modal parameters to establish comparisons.

The experimental means have been used for decades to observe the resonance modes of the guitar soundboards, alone, or when coupled with the sides and the remaining parts. As an example, it has been used for the objective characterisation of different guitar families, related to their soundboard braces pattern [3]. The experimental means are also useful for different purposes, such as the study of the global dynamics of the guitar and the radiated sound produced, dealing with a macro response of the instrument $[4,5]$. This type of response contains a high amount of information, and it becomes hard, considering the total coupling of all the elements, to attribute the role of each part of the guitar (and the properties of its components) with respect to the observed response.

Specific numerical models related to guitar have been developped since 70's [6], up to a detailed model in [7]. More recently, the evolution of the computational power enabled increasingly sophisticated models such as the complete channel of the production of sound, from the plucked guitar string to the radited sound [8]. One of the most complex model, mixing complete structure and fluid 
structure interactions has been developed in $[9,10]$ and enabled the computation of the radiated sound around the instrument. In [11], a detailed vibroacoustic model of a Portuguese guitar has been compared, in a deterministic way, with a real instrument. Usually, models of soundboards include braces, that have also been studied separately in [12]. In addition to the computation of the modal basis of guitar soundboards, the models have been used to compute the bridge admittance of the guitar, in a similar way than the one considered for violins, and thus provided results that could be compared with easily measurable features of real guitars [13].

So, historically, models were used as a posteriori, but, nowadays, increasingly for the prediction of complex structures. Physics-based modelling is used to predict the mechanical behaviour of complex virtual systems in the first steps of prototyping, and to quantify the variability of its behaviour, submitted to numerous unavoidable sources of uncertainties. Nevertheless, as the utilisation of the models concern more sophisticated structures, the models are still often unable to correctly predict their behaviour.

Thus, in order to avoid issues inherent to model prediction erros, the verification and validation $(\mathrm{V} \& \mathrm{~V})$ process has been developed to assess the viability of a model, and its framework is detailed in [14] and the book [15]. The V\&V method needs a large number of simulations to predict as much cases as possible, that are parts of the uncertainty domains. Its application on the vibratory behaviour of structures has been performed in $[16,17,18]$. The validation process aims at ensuring the reliability of the model when predicting the behaviour of a system. The predictions of the model (that can consist of numerous features) are compared to experimental data. The model is validated when the closeness between numerical and experimental results is below a tolerance level. The experiments make perfect sense when the material used exhibits a high variability, like the wood. Therefore, a higher number of experiments leads to an enhancement of the model reliability, through the validation criterion. 
This paper aims at highlighting in an innovative way for this application domain the potential of numerical models and their relevance in regard with musical acoustic applications. Indeed, in the framework of the utilisation of numerical models for the musical instrument making, the reliability of the models have to be at first time assessed and quantified, which is the main objective of this paper. In this study, a model validation of a musical instrument part is proposed. Experiments have been carried out on five similar Spanish guitar soundboards at 15 different steps $[19,20]$. Usually these steps performed by instrument makers are led by empiricism and traditions, and barely quantified to highlight objective assessments. In the case of wooden parts of musical instruments, the variability of such material is high and inevitable. Thus, this aleatory uncertainty is taken into account with stochastic approaches. Sensitivity analysis have to be performed to evaluate the relative influence of material parameters. Screened material parameters will be implemented with uncertainty model. The climatic conditions are also a source of uncertainty that is modelled with probabilistic approaches.

In the next section, the model development and methods used, as well as the parameters of the material behaviour's law are given. The results give dynamic data, sensitivity analysis results and dispersion of the computed eigenfrequencies with comparison with experimental ones. The conclusion give the main results and advances of this paper.

\section{Model and methods}

2.1. Experimental modal analysis of Spanish guitar soundboard

The modal analysis of the guitar soundboard is the same as the one performed in [19], the material and methods is based on this paper. The numerical 
model and experimental data used correspond to the construction stage № 15 of the paper [19], where all the braces and reinforcements are glued on the soundboard, and the rosette has been glued in its cavity. So, this stage corresponds to the state of the soundboard before being glued on the sides of the guitar. The five soundboards tested are labelled $S b_{01}, S b_{02}, S b_{03}, S b_{04}, S b_{05}$, and their masses are equal to $164,166,154,178$ and $170 \mathrm{~g}$, respectively. The average mass of the soundboards is equal to $166.4 \mathrm{~g}$. Only the out-of-plane motion of the soundboards has been considered. The guitar soundboards have been tested under free boundary conditions. A unidirectional accelerometer (B\&K 4518-003) has been used and glued near the future bridge location (at the point 69 on the figure 1 (a)). An impact hammer (B\&K 8204) has been used to excite the structure on the 99 points also displayed in the same figure. The test frequency ranges between 0 and $800 \mathrm{~Hz}$, and the corresponding resolution is equal to $0.25 \mathrm{~Hz}$. The vibration signals were measured and recorded as time series and processed into inertance FRF data. Both the applied excitation and the measured response were perpendicular to the soundboard. Signals were averaged two times for each measurement point. A modal analysis of the inertances has been performed to evaluate the modal basis of each soundboard. The modal analysis has led to the evaluation of a modal basis with eigenfrequencies and corresponding modal dampings for each modes. The experimental results obtained with this study, [19], for the first eight modes identified for the five soundboards are displayed in the table 3 .

\subsection{Computer aided design and meshing of the soundboard}

The numerical model has been developed using the finite element method based on a Computer Aided Design (CAD). The CAD software used is SOLIDWORKS ${ }^{\circledR}$. The software used for the pre-processing is PATRAN ${ }^{\circledR}$ and the solver is NASTRAN ${ }^{\circledR}$. The figure 1 (b) represents the CAD of the soundboard and the nomenclature. The figure 2 (a) displays the finite element model mesh. The CAD is meshed using tetrahedral elements with quadratic interpolation. The interfaces between the soundboard and the different parts are considered as perfect and are mo- 
delled with coincident nodes and equivalent faces. The finite element model contains 55000 elements and 104000 nodes and free boundary conditions are applied.

\subsection{Material orientation}

The material used is spruce, Picea abies, for all the braces, bars, patches and the soundboard. The material properties are taken from [21]. The values are given in the table 1 . The parts are oriented according to the figure 2 (a). The specific elastic parameters are sampled. The density $d_{0}$ is also sampled according to variations evaluated in [21]. The temperature and relative humidity are also sampled as equi-probalistic approaches bounded between 25 and $85 \%$ for $\mathrm{RH}$ and 15 to $35{ }^{\circ} \mathrm{C}$ for $\mathrm{T}$.

As a second step, the moisture content is calculated from the $\mathrm{RH}$ and $\mathrm{T}$ values sampled, according to [22], given in the eq. 1 :

$$
M C=10+0.16 \times(R H-50)-0.03 \times(T-21)
$$

The density as a function of $\mathrm{MC}, \rho_{M C}$, is then calculated from the value of $\mathrm{MC}$, according to [23], given in the eq. 2 :

$$
\rho_{M C}=\rho_{0} \times(1+0.01 \times(M C-10))
$$

The elastic constants values of $E_{L}, E_{R}$ and $G_{L R}$ depend on the relative humidity and, in a lesser degree, on the temperature. In order to implement this dependence, the values of the elastic properties are implemented as a function of $\mathrm{RH}$ and $\mathrm{T}$, laws are taken from [24], given in the eq. 3 :

$$
\begin{array}{r}
E_{L_{R H T}}=E_{L_{\rho}}(1-0.0015 \times(R H-50)-0.0008 \times(T-21)) \\
E_{R_{R H T}}=E_{R_{\rho}}(1-0.005 \times(R H-50)-0.0025 \times(T-21)) \\
G_{L R_{R H T}}=G_{L R_{\rho}}(1-0.007 \times(R H-50))
\end{array}
$$

When a set of elastic constants as a function of density ( $\frac{E_{i}}{\rho}$ as an example) is sampled, it is multiplied by a sampled value of density $\rho_{i}$. The value of $E_{i}$ is 
then modified following eq. 3, with sampled values of $\mathrm{RH}$ and $\mathrm{T}$. The sampled density is then also expressed as a function of the moisture content (eq. 2, given by 1 ), which gives $\rho_{M C_{i}}$. So, at the end of the sampling process, $E_{i}, G_{i j}$ and $\rho_{M C_{i}}$ are implemented in the numerical model, for the parts made of spruce.

Four sets of material parameters are considered dedicated to the soundboard, the braces, the bridge patch and the sound-hole reinforcement respectively. The material used for the parts is implemented to match the orientation of the wood in the reality. The wood samples are quarter-sawn and the dimensions are small enough to consider an orthotropic definition. Considering the coordinate frame represented in the figure $2(\mathrm{~b}), \mathrm{R}$ corresponds to the radial direction, $\mathrm{L}$ to the longitudinal direction, $\mathrm{T}$ to the tangential direction. A modal basis is computed with the nominal values given in the table 1 . With these values, the mass of the model is estimated to $168 \mathrm{~g}$ and the model average mass of the real soundboards is equal to $166.4 \mathrm{~g}$.

\subsection{Model-based modal analysis}

A modal analysis is computed with the numerical model. Starting at $1 \mathrm{~Hz}$, the first 50 modes are computed, which lead to a bandwidth for the modes extraction between 1 and $1000 \mathrm{~Hz}$. The frequency response functions (FRF) in acceleration, velocity and displacement (inertances, mobility and admittance), are computed on the 99 points of the experimental test, in the out-of-plane direction, Y. The driving force value is equal to $1 \mathrm{~N}$, applied in the same direction on the point labelled 69 in the figure 1 (a). The computation of the FRF is made on the numerical nodes close to each experimental points. In the considered frequency bandwidth, relative humidity and strain levels it is hypothesised that the material exhibits a linear elastic behaviour. Thus the damping is applied a posteriori as a modal damping whose value corresponds to the mean modal damping measured on guitar soundboards in the considered bandwidth, with $\xi=1.15 \%$. This value is taken from the experimental part and given in the table 3 . 


\subsection{Screening analysis}

Sensitivity analyses are computed to evaluate the impact of the variability of inputs of the model with regard to eigenfrequencies and eigenvectors outputs $Y\left(X_{i}\right)$ matched using MAC criterion [25]. Finite difference analysis is used at first to roughly screen the material and climtic parameters as a function of their impact. For this purpose, a variation $\delta X_{i}$ equal to $1 \%$ is applied on each input parameter $X_{i}$ one at a time. The sensitivity indicator is given by the eq. 4 [26] :

$$
\phi_{i}=\frac{\frac{Y\left(X_{1}, \ldots, X_{i}+\Delta X_{i}, \ldots, X_{n}\right)-Y\left(X_{1}, \ldots, X_{i}, \ldots, X_{n}\right)}{Y\left(X_{1}, \ldots, X_{i}, \ldots, X_{n}\right)}}{\frac{\Delta X_{i}}{X_{i}}}
$$

The morris screening analysis [27] is used to explore the input domain. The linear and coupling effects of the $X_{i}$ are evaluated. For a number of $n_{p}$ parameters $X_{i}$ in a $n_{p}$ dimensions domain $\Omega$, the domain is sampled in $l$ levels. The values of the domain $\Omega$ are defined in order to depict all the values that the parameters can attain. The elementary effect of a parameter $X_{i}$ in a sample $X^{j}$ of the space is given by :

$$
E_{i}^{j}=\frac{f\left(X^{j} \pm e_{i}\right)-f\left(X^{j}\right)}{ \pm \Delta}
$$

With $e_{i}$ a unit vector and $\Delta$ a value taken in $\left\{\frac{1}{p-1}, \ldots, 1-\frac{1}{p-1}\right\}$. The $n_{t}$ trajectories will define the number of computations given by $\left(n_{p}+1\right) \times\left(n_{t}\right)+1$. For this computation, the number of levels is equal to $6, n_{t}$ is equal to 20 and $n_{p}$ is equal to 12 after finite difference analysis first screening. This lead to 261 computations.

\subsection{Stochastic analysis}

The uncertainty quantification is performed using Monte-Carlo sampling method. For the uncertainty quantification, 750 modal bases are computed. The comparison of the matched eigenmodes between nominal model results and the ones obtained with modified input parameters is performed. Material and climatic parameters are defined as equiprobabilistic distributions between upper and lower values given in the table 1 . The matched eigenfrequencies are given as box 
and whiskers plots and the experimental data are compared with the computed distributions. The error between numerical eigenfrequencies and averaged measured eigenfrequencies is compared with the relative standard deviation (RSD) of each numerical eigenfrequencies.

\section{7. $C F D A C$}

A frequency domain assurance criterion is used, based on the experimental and computed FRFs. This method can be regarded as the equivalent of the Modal Assurance Criterion in the FRF domain. The FDAC [28], adapted as Complex-FDAC, is expressed in the eq. 6 [29] :

$$
C F D A C_{f g}=\frac{\left[\sum_{i=1}^{N} \sum_{j=1}^{N} h_{i j}\left(\omega_{f}\right) h_{i j}^{d}\left(\omega_{g}\right)\right]^{2}}{\left[\sum_{i=1}^{N} \sum_{j=1}^{N} h_{i j}\left(\omega_{f}\right) h_{i j}\left(\omega_{f}\right)\right]\left[\sum_{i=1}^{N} \sum_{j=1}^{N} h_{i j}^{d}\left(\omega_{g}\right) h_{i j}^{d}\left(\omega_{g}\right)\right]}
$$

In this equation, $i$ and $j$ correspond to the excitation and measure FRF, $h_{i j}$ refers to pristine state FRFs, $h_{i j}^{d}$ refers to altered FRFs. $f$ and $g$ refer to each pair of spectral lines compared from the two sets of mobility functions. $\mathrm{N}$ is the number of sampled points in the specimen. CFDAC results in a complex twodimensional array of dimension $\mathrm{N} \times \mathrm{N}$. Real and imaginary parts of the CFDAC are absolute-valued even when used for numeric computations. The CFDAC is performed between real soundboards and model nominal cases to observe the discrepancies in the behaviour of the soundboards.

\subsection{Fuzzy-FRF analysis}

The Fuzzy frequency response function (FUZZY-FRF) is computed using the $750 \mathrm{FRF}$ that are computed during the uncertainty quantification process. For each case, admittance is computed. The FRF used are located at the same point than experimental ones. The $750 \mathrm{FRF}$ are gathered in the same plot, and space of the plot is discretised in $2500 \times 2500$ subspaces. In each subspace, the number of lines passing through the subspace is evaluated. A color scale is applied to represent the amount of lines per subspace. This method is taken from [30, 31]. 


\subsection{Modal overlap factor}

The modal overlap factors $(M O F)$ by third octave bands are calculated using the eq 7

$$
M O F=M_{d} \times \eta \times F_{c}
$$

With $M_{d}$ the modal density, given by the ratio between the number of modes per third octave bands, for a central frequency $F_{c} . \eta$ is the loss factor of the system in the considered bandwidth and is equal to $2.3 \%$, two times the average value of the modal damping, given in the table 3. Only one domain is considered, the low-frequencies where the MOF value is comprised between 0 and $30 \%$.

\section{Results}

In this section the experimental and numerical results are given in different subsections.

\subsection{Experimental and numerical deformed shapes}

The measures on the five soundboards have led to five experimental modal bases. The values of the first eight eigenmodes frequencies and corresponding modal dampings are given in the table 3 . The first eight numerical eigenmodes shapes and nominal eigenfrequencies are given in the figure 4 . The eigenmodes shape consist in torsion modes in the LR plane, flexure modes in the L and R directions of the soundboard and mixed torsion and flexure modes. The modal overlap factor values are given in the table 2. Below $500 \mathrm{~Hz}$, the modal overlap factor is comprised between 2 and $28 \%$ which corresponds to the low frequency domain, where, mainly, the modal analysis is relevant. Above $500 \mathrm{~Hz}$ the mid-frequencies domain is reached, which suggests an increase of discrepancies between experimental and numerical results. 


\subsection{Co-located nominal FRF comparison}

The co-located FRF in acceleration of the nominal model and the experimental soundboards are given in the figure 3 . It is shown that the level of the dynamical responses of the experimental soundboards are close to the computed one up, and it is not possible to differentiate them based on the acceleration level.

\subsection{Comparison between numerical and experimental eigenfrequencies, deter- ministic approach}

The eigenfrequencies, as well as their mean and standard deviation, for the first eight modes are given in the table 3 . The average relative standard deviation (RSD) of the experimental eigenfrequencies is equal to $4.3 \%$ and the average modal damping is equal to $1.15 \%$ on the modes considered. The relative error between nominal model eigenfrequencies and experimental ones is comprised between -6.3 and $7.2 \%$.

\subsection{Spectral correlation of numerical and experimental guitar soundboard}

The figure 5 shows the frequency domain assurance criterion from 0 to 800 $\mathrm{Hz}$ for each case. For the first four sboundboard, up to $600 \mathrm{~Hz}$, the CFDACs show a good correlation between experimental and numerical databases, which corresponds to the domain of low-frequencies, as shown in the table 2. Moreover, the CFDAC matrix complex correlation is almost diagonal, which indicates a good correspondence between stiffness and mass of the model and the real soundboards.

\subsection{Stochastic analysis results}

The results of the uncertainty quantification study are given for the first eight modes as box and whiskers plots on the figure 6 and mean, SD and RSD in the table 4 . In the figure 6 , the in boxes vertical lines correspond to the median, the lower and upper limits of the boxes correspond to the lower and upper first quartile (25 percentiles) respectively and the limits of the left and right 
whiskers correspond to 9 and 91 percentiles respectively. It is shown that all the experimental eigenfrequencies are comprised between the 9 and 91 percentiles. Moreover, most of the experimental eigenfrequencies are comprised in the second quartile (50 percentiles).

In the table 4 , it is shown that the RSD of the first eight numerical eigenfrequencies is rather diffuse and comprised between 5.8 and $8.2 \%$, with an average value equal to $6.9 \%$.

\subsection{Fuzzy-FRF of a free edges soundboard of Spanish guitar soundboard}

The figure 7 (a) shows the Fuzzy-FRF evaluated with the database used for the uncertainty quantification, which corresponds to the co-located FRF in displacement of the 750 computations. This figure gives a display of the variability distribution of the FRF of the studied structures when undergoing material and climatic variations. The figure 7 (a) displays the number of FRF that are comprised in each discretised subspace of the plot. Each axis is divided 2500 times, which means that the figure displays $6.25 \times 10^{6}$ discretised squares. So, the sampling is equal to $0.32 \mathrm{~Hz}$ for the frequency axis and from $5.10^{-3}$ to $40 \mathrm{~m} . \mathrm{s}^{-2} . \mathrm{N}^{-1}$ for the acceleration axis. The maximum number of FRF inside a square is equal to 100, at the lowest frequency where the dispersion is the lowest. This value is used as the maximum value for the color fringe and the lowest value 0 corresponds to the case where no FRF crosses a discretised square. The dispersion of the FRF increases above $100 \mathrm{~Hz}$, and becomes rather diffuse above this value. The figure 7 (b) gives the min and max values of the FRF, represented as dashed black lines. The experimental FRF are also displayed in the figure 7 (b), and are generally comprised inside the area of presence probability, where the number of occurrences is higher than 30 .

\subsection{Screening analyses results}

The ranking of the elementary effects of the material and climatic parameters is given for each modes in the figure 4. Depending on the eigenmode shape, the influential material parameters ranking varies, and is in accordance with the 
finite differences sensitivity matrix given in the figure 8 (a). As a primary result of the sensitivity matrix, it is shown that the specific elastic parameters of the soundboard are the most influential. The specific elastic parameters, when their complete range is considered are more influential on the eigenfrequencies than the densities. The relative humidity impact on the eigenfrequencies is almost constant for each mode and corresponds to values comprised between 14 and 18 $\%$ of the total of the elementary effects.

The ranking of the material and climatic parameters for global dynamical behaviour of the first 20 modes is given in the figure 8 (a) for the eigenfrequencies and (b) for the eigenvectors. It is shown that the influence of each parameter varies according to the dynamical feature observed. Generally, in free conditions, the specific rigidity in the longitudinal direction of the soundboard plate is the most influential parameter (22\% of the total), followed by the specific shear rigidity in LR plane (19\%) and the specific radial rigidity (15\%). The impact of the density of the soundboard is similar to the impact of the density of the braces (9 and $8 \%$, respectively). The remaining specific elastic parameters of the braces are less influential, and correspond to $7 \%$ for the braces $\mathrm{L}$ rigidity and $3 \%$ for the LR shear elasticity of the braces. The RH has a strong impact on the eigenfrequencies, corresponding to $17 \%$ of the total elementary effects. The effect of the $\mathrm{RH}$ is smaller on the eigenvectors; as its effect is global on every elastic and density parameter, it affects in a smaller way the eigenmode shapes. In has to be pointed out that, considering eigenvectors, the longitudinal specific modulus of the soundboard is mainly influential, up to two times the specific rigidities in $\mathrm{R}$ direction or $\mathrm{LR}$ plane.

\section{Discussion}

The results obtained lead to multiple discussions. First of all, the deterministic comparison has highlighted the good predictive capability of the model concerning the dynamical behaviour of Spanish guitar soundboards. It has been shown that the relative error between a nominal model (whose material and 
climatic parameters are taken form the literature) and the average values of eigenfrequencies is close to $4 \%$, which is close to the relative standard deviation of the experimental eigenfrequencies. When material and climatic uncertainties are taken into account, the model is able to predict every experimental frequencies, which are, in addition, comprised in the second quartile (50 percentiles) of the computed ones. The comparison of deterministic numerical and experimental FRF have shown that the discrepancies increase above $500 \mathrm{~Hz}$ which is close to the limit of the low frequencies domain given by the modal overlap factor. This result is in accordance with dynamics theory, and highlights the limits of a modal point of view for this type of study. The CFDAC comparison between experimental and numerical FRF is innovative in this application domain, enables a global comparison and is more adapted in mid-frequencies range. The CFDAC correlation criterion is a relevant quantified global indicator of closeness between numerical and experimental data. The Fuzzy-FRF feature proposed here shows the dispersion of the model response for a fixed design. Thus, a guitar soundboard built with the same geometry and the same wood species will exhibit a dynamical behaviour that may be included in the FuzzFRF prediction. As shown in this study, experimental FRF were comprised in the fuzzy-FRF high plausibility area and inside the upper and lower bounds of the stochastic simulations, as shown in the figure 7 (b). This post-processing of the FRF in the case of an uncertainty quantification can be a useful tool for the decision-support in musical instruments making, and to confirm the relevance of a design change over the material and climatic variability impact.

Based on the fact that the model was able to correctly predict dynamical behaviour, it has been shown that the most influential parameters with regard to the eigenfrequencies and eigenvectors of a free conditions soundboard were mainly specific elastic parameters $\frac{E_{L}}{\rho}, \frac{E_{R}}{\rho}$ and $\frac{G_{L R}}{\rho}$ of the top plate soundboard, followed by the relative humidity and the density of the plate and braces. These results are similar to the one obtained considering a violin in [32]. It has been shown in [33] and [21] that studies on tonewood were most of the time focused on $\frac{E_{L}}{\rho}$, which is also correlated with high "quality" wood. Considering these new 
results, it is clear that, as $\frac{E_{R}}{\rho}$ and $\frac{G_{L R}}{\rho}$ play an important role, the selection criteria and studies should also focus on these parameters.

\section{Conclusion}

In this paper, the comparison between experimental and numerical dynamical data have shown the capability of physic-based models to predict complex assemblies responses. It has been shown that model accuracy was maintained even in the presence of strong material and climatic uncertainties. This paper proposes new ways for the characterisation of musical instruments : the uncertainty quantification for a given geometry, the CFDAC between model and real instruments and the Fuzzy-FRF for the post processing of the study of the uncertainties effects on the dynamical behaviour of musical instruments. This is a first and innovative step in the validation process of physics-based models of musical instruments, which, associated with the different dynamical features proposed for this domain can be used for design and restoration purposes of musical instruments. Moreover, the results obtained have questioned the current selection criterion for spruce tonewood, and shown that specific moduli other than $\frac{E_{L}}{\rho}$ were also significant on the dynamics of the guitar soundboard in free conditions, which is its main boundary conditions during making steps. These results are relevant in the low frequency domain, where modal analysis is effective. In order to manage with higher domains, such as mid-frequencies and high frequencies domains, large frequency bandwidth descriptors [34], such as mean-values approaches should be considered, and have already proved useful for acoustic guitars [13], violins [35] and composite plates [36]. Modal approaches and medium and high frequencies methods would provide, through model validation process, reliable large band datasets of musical instruments behaviours, even in presence of strong uncertainties. 


\section{Acknowledgements}

Funding : This work has been performed in the Framework of EUR EIPHI (ANR-17-EURE-0002).

\section{References}

[1] E. Skrodzka, A. Lapa, B. B. J. Linde, E. Rosenfeld, Modal parameters of two incomplete and complete guitars differing in the bracing pattern of the soundboard, The Journal of the Acoustical Society of America 130 (4) (2011) 2186-2194.

[2] J. A. Torres, R. R. Boullosa, Influence of the bridge on the vibrations of the top plate of a classical guitar, Applied Acoustics 70 (11-12) (2009) 13711377. doi :10.1016/j.apacoust.2009.07.002.

URL http://dx.doi.org/10.1016/j.apacoust.2009.07.002

[3] G. Caldersmith, Designing a guitar family, Applied Acoustics 46 (1) (1995) 3-17. doi :10.1016/0003-682X(95)93949-I.

[4] I. Perry, Sound Radiation Measuremets on Guitars and Other Stringed Musical Instruments, Ph.D. thesis, Cardiff University (2014).

[5] B. Richardson, Guitar making-the acoustician's tale, in : Proc. Second Vienna Talk, Vienna, 2010, pp. 125-128.

URL http://www. laguitarra-blog.com/wpcontent/uploads/2011/08/guitarmaking.pdf

[6] H. L. Schwab, K. C. Chen, Finite element analysis of a guitar soundboard, Catgut Acoust. Soc. Newsletter 24 (1975) 13-15.

[7] A. Chaigne, Numerical simulations of stringed instruments-today's situation and trends for the future, Catgut Acoustical Society Journal 4 (5) (2002) 12-20. 
[8] E. Bécache, A. Chaigne, G. Derveaux, P. Joly, Numerical simulation of a guitar, Computers and Structures 83 (2-3) (2005) 107-126. doi :10.1016/B978-008044046-0.50305-5.

URL http://www.sciencedirect.com/science/article/pii/S0045794904002974

[9] G. Derveaux, Modélisation numérique de la guitare acoustique, Ph.D. thesis, École Polytechnique (2002).

URL papers://8125aba6-5c2a-4362-9a18-0ed7f2b10f00/Paper/p4776

[10] G. Derveaux, A. Chaigne, P. Joly, E. Bécache, Time-domain simulation of a guitar : Model and method, The Journal of the Acoustical Society of America 114 (6) (2003) 3368-3383. doi :10.1121/1.1629302.

[11] M. Vieira, V. Infante, P. Serrão, A. M. Ribeiro, Experimentalnumerical correlation of the dynamic behavior of the Portuguese guitar, Applied Acoustics 131 (September 2017) (2018) 51-60. doi : 10.1016/j.apacoust.2017.10.007.

URL https://doi.org/10.1016/j. apacoust.2017.10.007

[12] J. Bretos, C. Santamari, J. Alonso Moral, Finite element analysis and experimental measurements of natural eigenmodes and random responses of wooden bars used in musical instruments, Applied Acoustics 56 (3) (1999) 141-156. doi :10.1016/S0003-682X(98)00030-9.

URL http://www.sciencedirect.com/science/article/pii/S0003682X98000309

[13] B. Elie, F. Gautier, B. David, Macro parameters describing the mechanical behavior of classical guitars, The Journal of the Acoustical Society of America 132 (6) (2012) 4013-4024. doi :10.1121/1.4765077.

URL http://asa.scitation.org/doi/10.1121/1.4765077 http://www.ncbi.nlm.nih.gov/pubmed/23231130

[14] ASME, AIAA Guide for the Verification and Validation of Computational Fluid Dynamics Simuations (G-077-1998) (1998). 
[15] W. L. Oberkampf, C. J. Roy, Verification and validation in scientific computing, Cambridge University Press, 2010.

[16] F. Hemez, Uncertainty quantification and the verification and validation of computational models, Damage Prognosis for Aerospace, Civil and Mechanical Systems (2004) 201-220.

[17] F. Hemez, S. Atamturktur, C. Unal, Defining predictive maturity for validated numerical simulations, Computers and Structures 88 (7-8) (2010) 497-505. doi :10.1016/j.compstruc.2010.01.005.

URL http://dx.doi.org/10.1016/j.compstruc.2010.01.005

[18] B. H. Thacker, S. W. Doebling, F. Hemez, M. C. Anderson, J. E. Pepin, E. A. Rodriguez, Concepts of model verification and validation, Tech. rep., Los Alamos National Lab., Los Alamos, NM (US) (2004).

[19] M. A. Pérez, A frequency domain correlation approach for the assessment of wooden musical instruments, in : Analysis and Characterisation of Wooden Cultural Heritage by means of Scientific Engineering Methods, no. April 2016, Halle/Saale, 2016, pp. 77-83.

URL https://www.researchgate.net/publication/338459211_A_frequency_domain_correlation_a

[20] M. A. Pérez, R. Serra-lópez, A Frequency Domain Correlation Approach for Musical Instruments Experimental Assessment, in : IMAC-XXXV Conference and Exposition on Structural Dynamics, no. January 2017, Orange county, 2017.

URL https://www.researchgate.net/publication/338459213_A_Frequency_Domain_Correlation_A

[21] R. Viala, V. Placet, S. Cogan, Simultaneous non-destructive identification of multiple elastic and damping properties of spruce tonewood to improve grading - in press, Journal of Cultural Heritagedoi :10.1016/j.culher.2019.09.004.

URL https://doi.org/10.1016/j.culher.2019.09.004 
[22] W. T. Simpson, Predicting equilibrium moisture content of wood by mathematical models, Wood and Fiber 5 (1) (1973) 41-49.

URL http://www.bcin.ca/Interface/openbcin. cgi?submit=submit\&Chinkey=52402

[23] W. T. Simpson, Specific Gravity, Moisture Content, and Density Relationship for Wood, Tech. rep., United States Department of Agriculture (1976). URL http://citeseerx.ist.psu.edu/viewdoc/download?doi=10.1.1.155.4926\&rep=rep1\&type=pdf

[24] R. Viala, Towards a model-based decision support tool for stringed musical instrument making, Ph.D. thesis, Université Bourgogne Franche-comté (2018).

URL http://www.theses.fr/2018UBFCD002

[25] R. J. Allemang, D. L. Brown, A correlation coefficient for modal vector analysis, in : First International Modal Analysis Conference, Orlando, 1982, pp. $110-116$.

[26] D. M. Hamby, A review of techniques for parameter sensitivity analysis of environmental models, Environmental Monitoring and Assessment 32 (2) (1994) 135-154. doi :10.1007/BF00547132.

[27] M. D. Morris, Factorial Sampling Plans for Preliminary Computational Experiments, Technometrics 33 (2) (1991) 161-174. arXiv :arXiv :1011.1669v3, doi :10.2307/1269043.

[28] R. Pascual, J. C. Golinval, M. Razeto, A Frequency Domain Correlation Technique for Model Correlation and Updating, in : 15th International Modal Analysis Conference (IMAC XV), Orlando, 1997, pp. 587-592. arXiv :arXiv :1011.1669v3, doi :10.1017/CBO9781107415324.004.

URL http://hdl.handle.net/2268/25147

[29] M. A. Pérez, R. Serra-López, A frequency domain-based correlation approach for structural assessment and damage identification, Mechanical Systems and Signal Processing 119 (2019) 432-456. doi :10.1016/j.ymssp.2018.09.042. 
[30] D. Moens, D. Vandepitte, Interval Uncertainty Quantification in Numerical Models using Dynamic Fuzzy Finite Element Analysis, in : NATO AVT147 Symposium on Computational Uncertainty in Military Vehicle Design, Neuilly-sur-Seine, 2007, pp. 1-16.

[31] M. De Munck, D. Moens, W. Desmet, D. Vandepitte, A fuzzy FRF analysis of a stiffened conical shell structure using an intelligent Kriging based optimisation procedure, in : 50th AIAA/ASME/ASCE/AHS/ASC Structures, Structural Dynamics, and Materials Conference 17th AIAA/ASME/AHS Adaptive Structures Conference 11th AIAA, 2009, p. 2301.

URL http://www.scopus.com/inward/record.url?eid=2-s2.084855620470\&partnerID=40\&md5=3ad6d1c4154e98611811751 $\mathrm{fe} 9 \mathrm{~b} 00942$

[32] R. Viala, V. Placet, S. Cogan, E. Foltête, Model-based effects screening of stringed instruments, in : Conference Proceedings of the Society for Experimental Mechanics Series, Vol. 3, 2016, pp. 151-157. doi :10.1007/978-3319-29754-514.

[33] C. Carlier, A. Alkadri, J. Gril, I. Brémaud, Revisiting the notion of " resonance wood " choice : a decompartementalised approach from violin makers' opinion and perception to characterization of material properties ' variability, in : Wooden musical instruments - Different forms of knowledge : Book of end of WoodMusICK COST Action FP1302, 2018.

[34] J. Woodhouse, Plucked guitar transients : Comparison of measurements and synthesis, Acta Acustica united with Acustica 90 (5) (2004) 945-965.

[35] B. Elie, B. David, Analysis of bridge mobility of violins, in : Proceedings of the Stockholm Music Acoustics Conference, SMAC 2013, Vol. 6, Stockholm, 2013, pp. 54-59.

URL http://hal.inria.fr/hal-01060528/

[36] K. Ege, N. B. Roozen, Q. Leclère, R. G. Rinaldi, Assessment of the apparent bending stiffness and damping of multilayer plates; modelling and 
experiment, Journal of Sound and Vibration 426 (May) (2018) 129-149. doi :10.1016/j.jsv.2018.04.013.

[37] D. Guitard, F. El Amri, Modèles prévisionnels de comportement élastique tridimensionnel pour les bois feuillus et les bois résineux, Annales des sciences forestières 44 (3) (1987) 335-358.

[38] I. Brémaud, J. Gril, B. Thibaut, Anisotropy of wood vibrational properties : Dependence on grain angle and review of literature data, Wood Science and Technology 45 (4) (2011) 735-754. doi :10.1007/s00226-010-0393-8. 
Figure 1: (a) location of the experimental measurement points and FRF synthesis nodes used in [19] experiments; (b), Computer Aided Design and nomenclature of Spanish guitar soundboard.

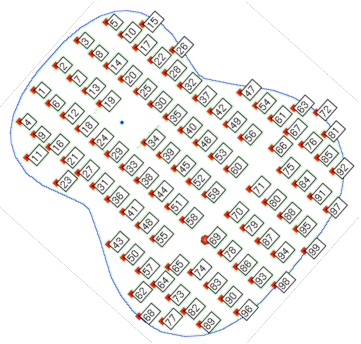

(a)

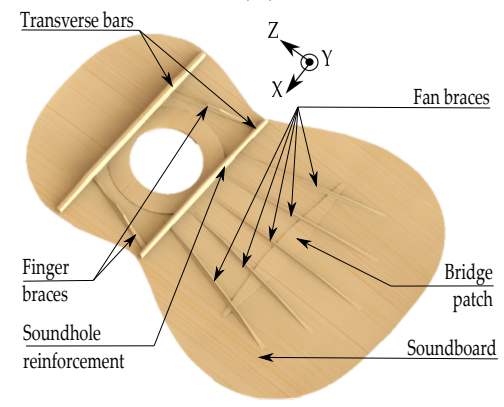

(b) 
FIgURE 2: (a) finite element model of the guitar soundboard; (b) orientation of the material, $\mathrm{L}$ : longitudinal, $\mathrm{R}$ : radial, $\mathrm{T}$ : tangential.

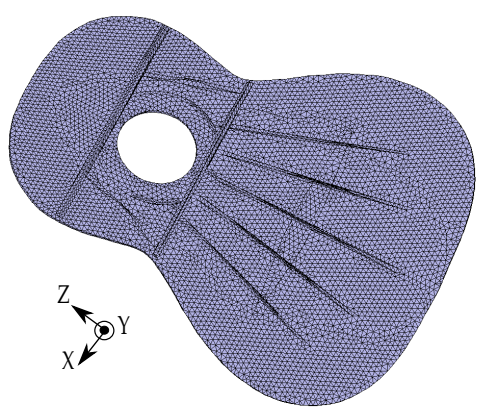

(a)

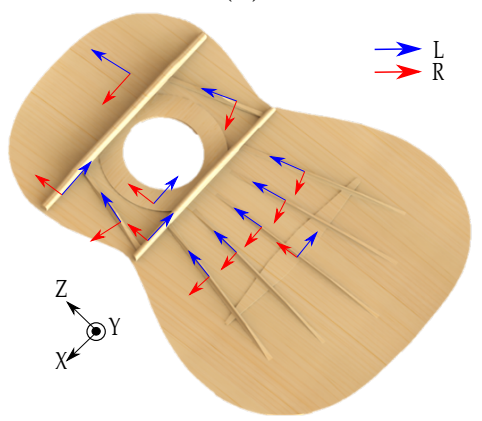

(b)

FIGURE 3: Inertances of the colocated measured and excitation point for both model and experimental FRF.

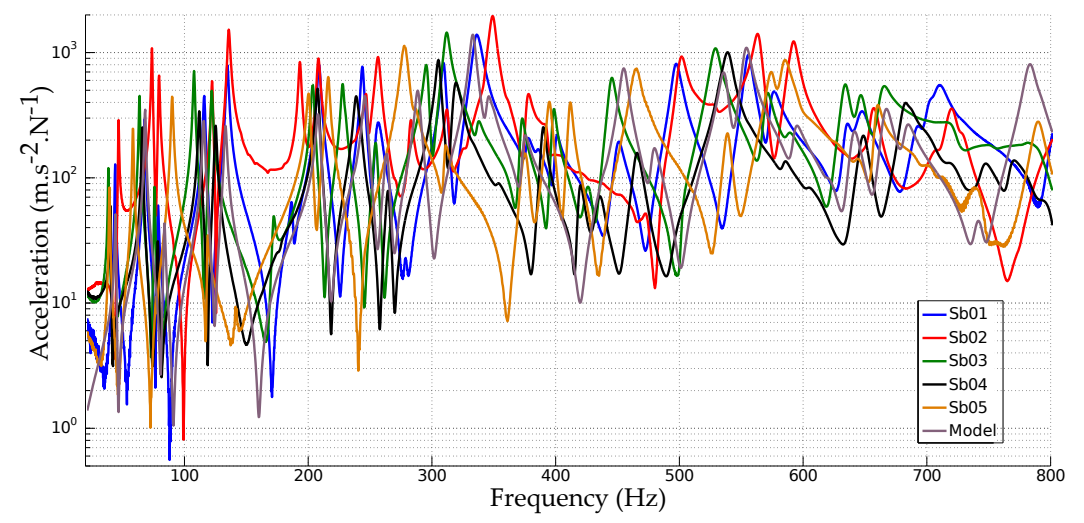


TABLE 1: Material properties of spruce implemented in the numerical model; italic from [21] at $M C=10 \%$. Remaining values from [37] and [38]. Loss factors values are given but have not been implemented for undamped modal analysis.

\begin{tabular}{cccc} 
Parameter & Mean value & Min. value & Max. value \\
\hline$\frac{E_{L}}{\rho}\left(\mathrm{MPag}^{-1} \mathrm{~cm}^{-3}\right)$ & 29000 & 20590 & 35380 \\
$\frac{E_{R}}{\rho}\left(\mathrm{MPag}^{-1} \mathrm{~cm}^{-3}\right)$ & 2280 & 1460 & 3810 \\
$\frac{E_{T}}{\rho}\left(\mathrm{MPag}^{-1} \mathrm{~cm}^{-3}\right)$ & 1480 & 1300 & 1660 \\
$\nu_{L R}(-)$ & 0.37 & - & - \\
$\nu_{R T}(-)$ & 0.48 & - & - \\
$\nu_{T L}(-)$ & 0.02 & - & - \\
$\eta_{L}(\%)$ & 0.73 & 0.09 & 0.12 \\
$\eta_{R}(\%)$ & 1.7 & 0.17 & 0.1 \\
$\eta_{L R}(\%)$ & 1.2 & 0.2 & 0.17 \\
$\frac{G_{L R}}{\rho}\left(\mathrm{MPag}^{-1} \mathrm{~cm}^{-3}\right)$ & 1850 & 1295 & 2442 \\
$\frac{G_{R T}}{\rho}\left(\mathrm{MPag}^{-1} \mathrm{~cm}^{-3}\right)$ & 100 & 74 & 150 \\
$\frac{G_{T L}}{\rho}\left(\mathrm{MPag}^{-1} \mathrm{~cm}^{-3}\right)$ & 1910 & 1070 & 2750 \\
Density $\left(\mathrm{g} \mathrm{cm}^{-3}\right)$ & 0.44 & 0.39 & 0.51 \\
Relative humidity $(\%)$ & 50 & 20 & 85 \\
Temperature $\left({ }^{\circ} \mathrm{C}\right)$ & 21 & 15 & 35 \\
\hline
\end{tabular}

TABLE 2: Values of modal overlap factor for corresponding third octaves bands.

\begin{tabular}{ccc} 
Third octave band & M.O.F. (\%) & Domain \\
\hline 40 & 8 & L.F. \\
50 & 2 & L.F. \\
63 & 2 & L.F. \\
80 & 14 & L.F. \\
100 & 4 & L.F. \\
125 & 10 & L.F. \\
160 & 7 & L.F. \\
200 & 9 & L.F. \\
250 & 18 & L.F. \\
315 & 28 & L.F. \\
400 & 22 & L.F. \\
500 & 34 & M.F. \\
630 & 52 & M.F. \\
\hline
\end{tabular}


Figure 4: Left side, frequencies and deformed shapes of the first eight computed modes of the spanish guitar soundboard, in free-free conditions. The red color represents the highest eigenvectors values. Right side, elementary effect ranking for each parameter using Morris sensitivity Method.

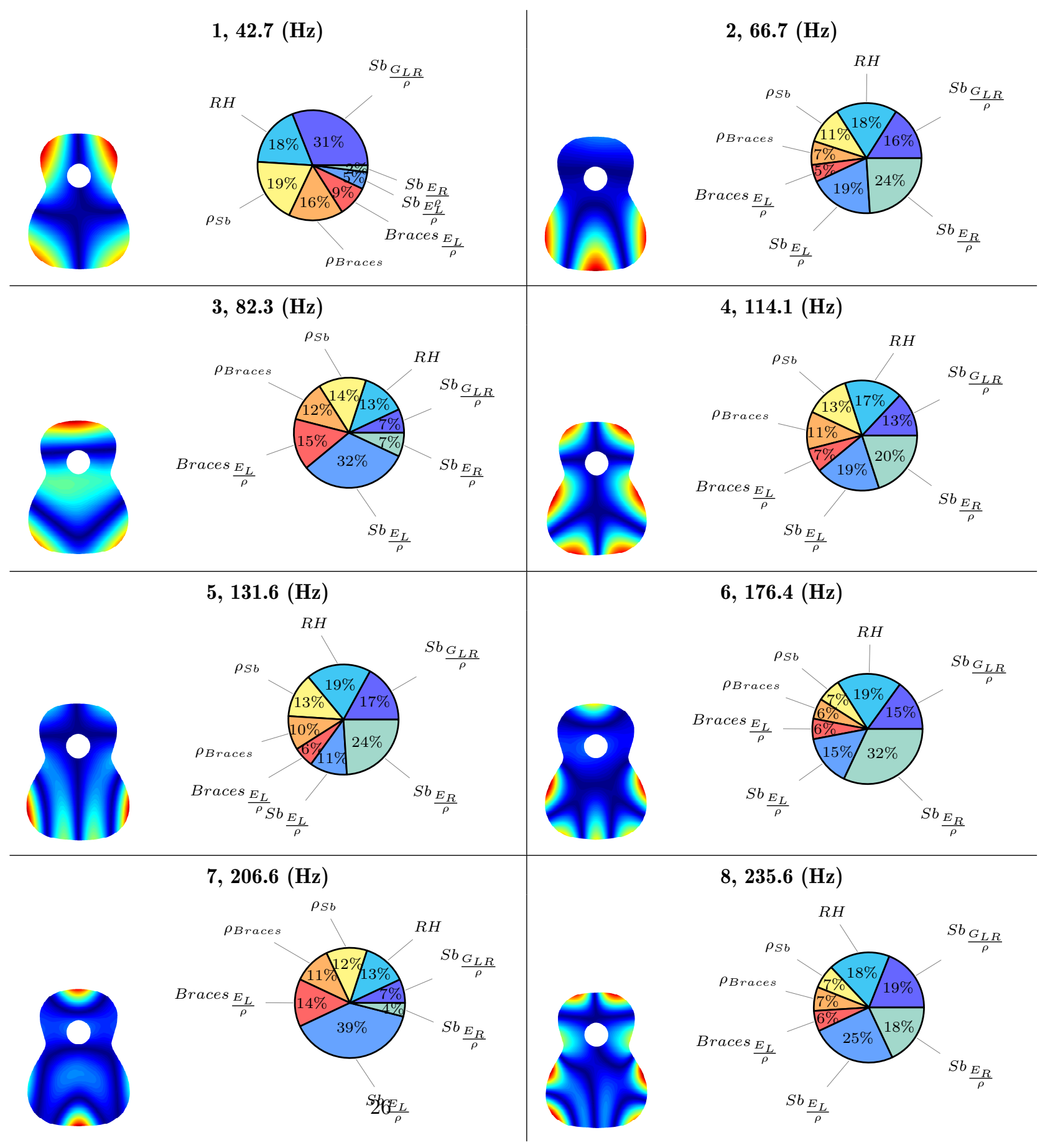


TABLE 3: Results for the first eight modes measured and computed. Mean, standard deivation (SD) and relative standard deviation (RSD) of the measured eigenfrequencies, as well as the average modal damping are given.

\begin{tabular}{|c|c|c|c|c|c|c|c|c|c|c|c|}
\hline Mode & $\begin{array}{l}\text { Num. } \\
(\mathrm{Hz})\end{array}$ & $\begin{array}{l}S b_{01} \\
(\mathrm{~Hz})\end{array}$ & $\begin{array}{l}S b_{02} \\
(\mathrm{~Hz})\end{array}$ & $\begin{array}{l}S b_{03} \\
(\mathrm{~Hz})\end{array}$ & $\begin{array}{l}S b_{04} \\
(\mathrm{~Hz})\end{array}$ & $\begin{array}{l}S b_{05} \\
(\mathrm{~Hz})\end{array}$ & $\begin{array}{c}\text { Av. exp } \\
(\mathrm{Hz})\end{array}$ & $\begin{array}{l}\text { SD. exp } \\
(\mathrm{Hz})\end{array}$ & $\begin{array}{c}\text { RSD. exp } \\
(\%)\end{array}$ & $\begin{array}{c}\text { Num. vs Exp. } \\
\text { error }(\%)\end{array}$ & Average $\xi(\%)$ \\
\hline 1 & 42.7 & 42.4 & 45.2 & 37 & 38.2 & 37.8 & 40.1 & 2.9 & 7.3 & 6.5 & 0.6 \\
\hline 2 & 66.7 & 66.3 & 72.3 & 62 & 64.5 & 56.9 & 64.4 & 4.0 & 6.1 & 3.6 & 0.7 \\
\hline 3 & 82.3 & 77.2 & 77.8 & 74.4 & 77 & 75.8 & 76.4 & 1.1 & 1.4 & 7.2 & 0.7 \\
\hline 4 & 114.1 & 114 & 120.9 & 106.2 & 110.5 & 117 & 113.7 & 4.3 & 3.8 & 0.3 & 1.1 \\
\hline 5 & 131.6 & 133 & 134 & 121 & 123 & 140 & 130.2 & 6.6 & 5.0 & 1.1 & 1.2 \\
\hline 6 & 176.4 & 185 & 192 & 170 & - & 199 & 186.5 & 9.0 & 4.8 & -5.7 & 1.3 \\
\hline 7 & 206.6 & 208 & 207 & 202 & - & 214 & 207.8 & 3.3 & 1.6 & -0.7 & 1.7 \\
\hline 8 & 235.6 & 242 & 245 & 253 & 237 & 275 & 250.4 & 10.9 & 4.3 & -6.3 & 2.0 \\
\hline Mean & - & - & - & - & - & - & - & 5.2 & 4.3 & 3.9 (abs.values) & 1.15 \\
\hline
\end{tabular}

TABLE 4: Mean, SD and RSD of the matched numerical eigenfrequencies.

\begin{tabular}{cccc} 
Mode & Mean $(\mathrm{Hz})$ & SD $(\mathrm{Hz})$ & RSD $(\%)$ \\
\hline 1 & 42 & 2.8 & 6.7 \\
2 & 66.6 & 4.9 & 7.3 \\
3 & 80.7 & 4.7 & 5.8 \\
4 & 112.8 & 7.7 & 6.8 \\
5 & 133.1 & 10.2 & 7.7 \\
6 & 177.1 & 14.6 & 8.2 \\
7 & 201.4 & 11.8 & 5.9 \\
8 & 233.6 & 6.3 & 7.0 \\
Mean & - & - & 6.9 \\
\hline
\end{tabular}


Figure 5: CFDAC matrix (magnitude) between the measured (ordonate) and computed with the nominal model (abscissa) FRF for each soundboard.

$S b_{01}$

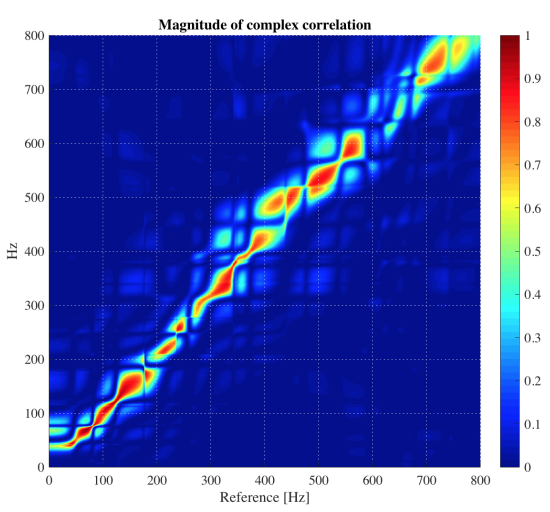

$S b_{03}$

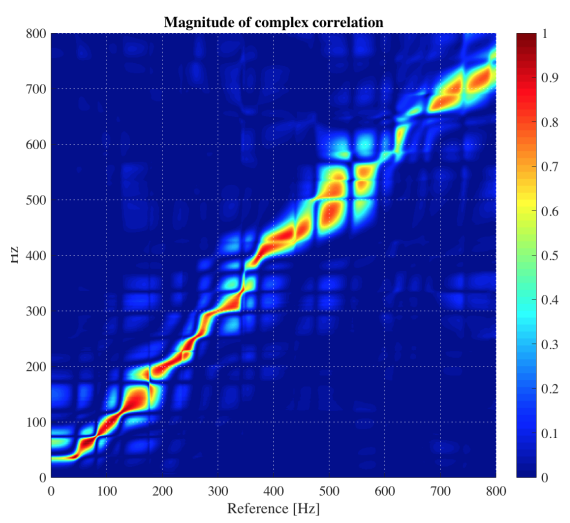

$S b_{02}$

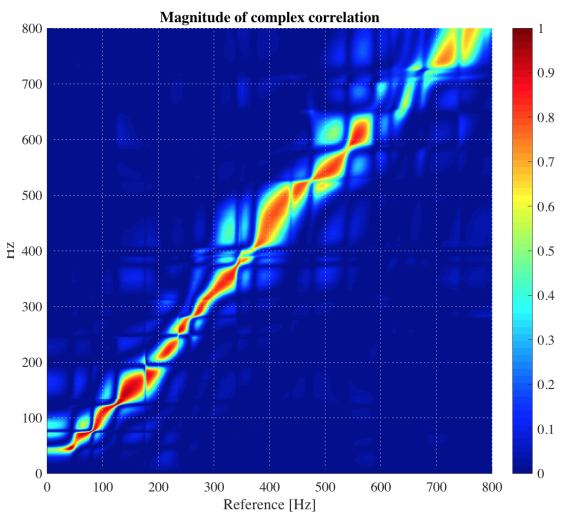

$S b_{04}$

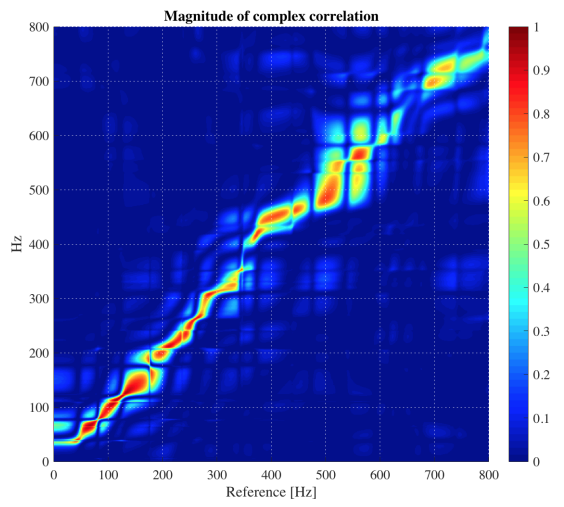

$S b_{05}$

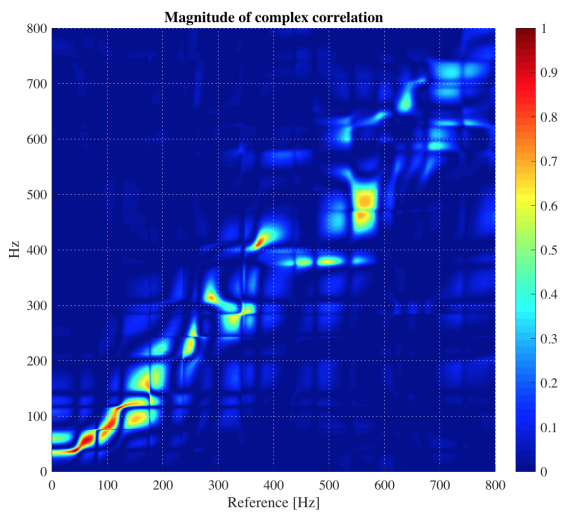


Figure 6: Box and whiskers plots of the values of the first eight matched eigenfrequencies computed by the numerical model of soundboard, comparison with experimental eigenfrequencies values. The vertical lines of the boxes correspond, from left to right, to the first quartile $\left(25^{\text {th }}\right.$ percentiles) the median and the third quartile. The limits of the left and right whiskers correspond to the $9^{t h}$ and $91^{t h}$ percentiles, respectively.

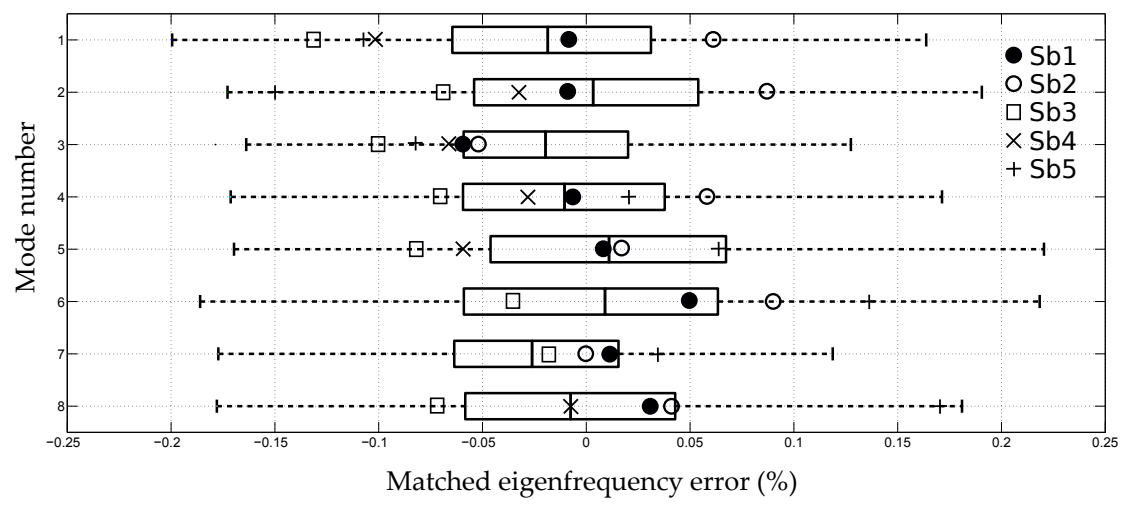


Figure 7: (a) fuzzy-FRF of the co-located admittances from the uncertainty quantification computations, the total number of runs is equal to 750 ; (b) statistical treatment of the FRFs, dashed black line, upper and lower limits of the stochastic computations.

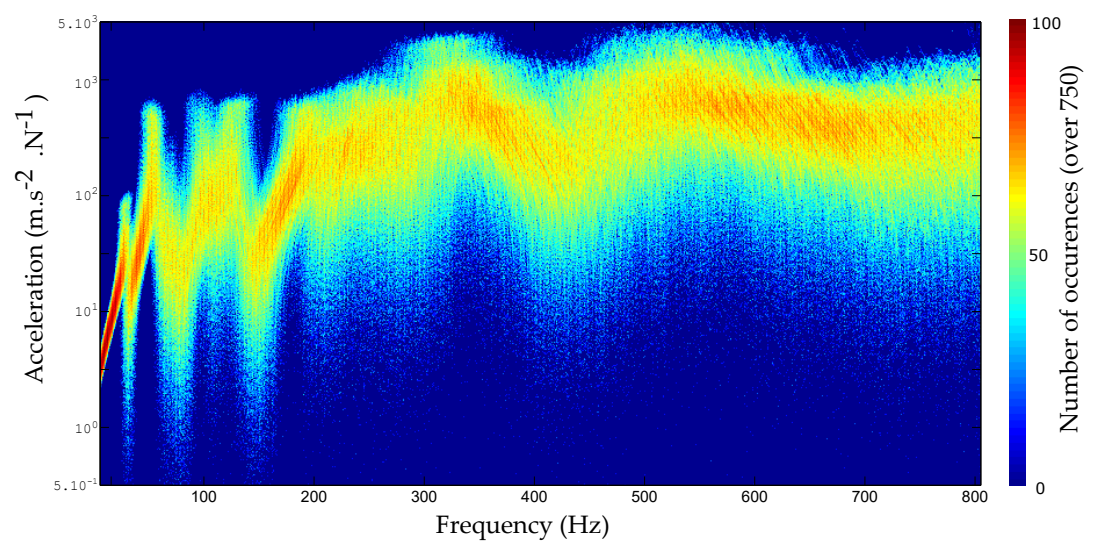

(a)

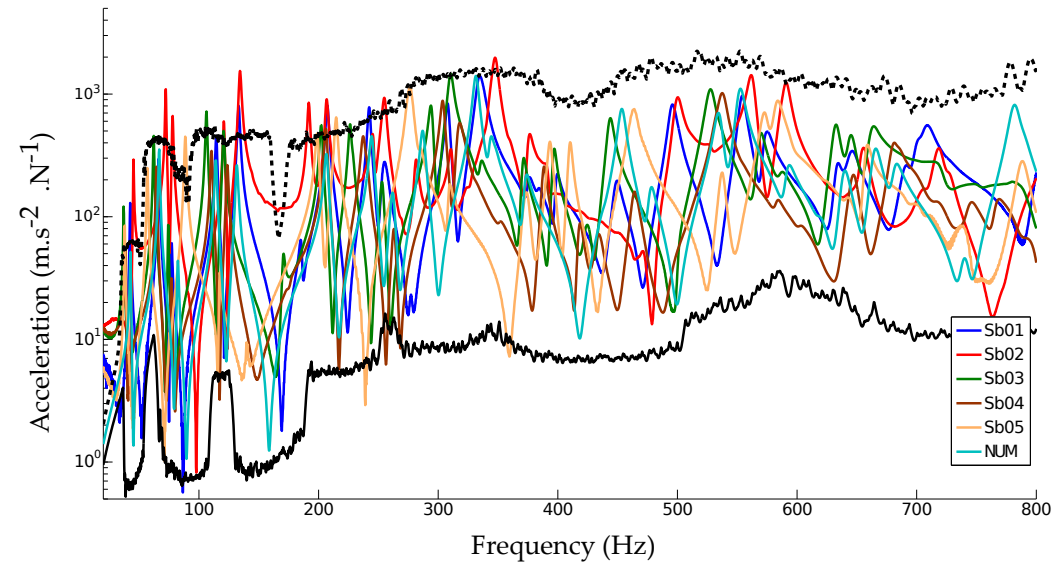

(b) 
Figure 8: Finite difference sensitivity matrix for the first 30 modes (a); relative elementary effects of the material and climatic parameters in regard with eigenfrequencies (b) and eigenvectors (c).

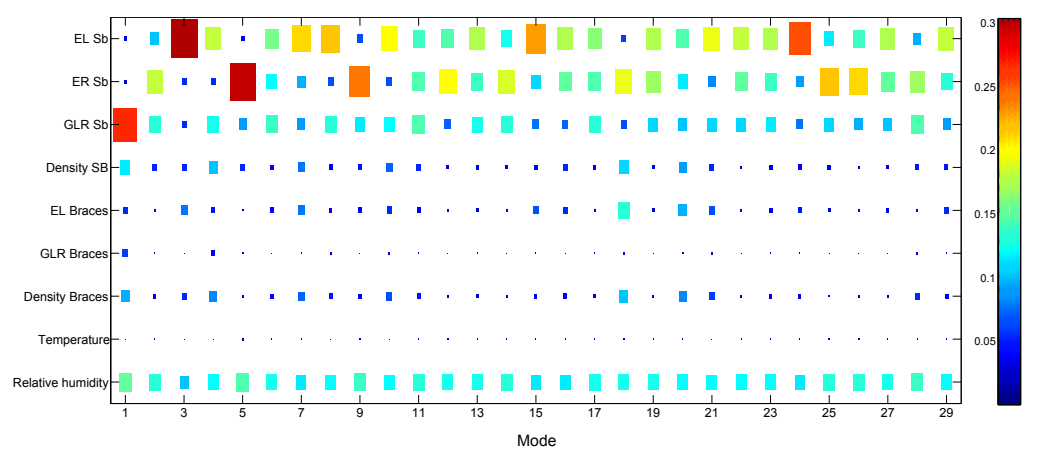

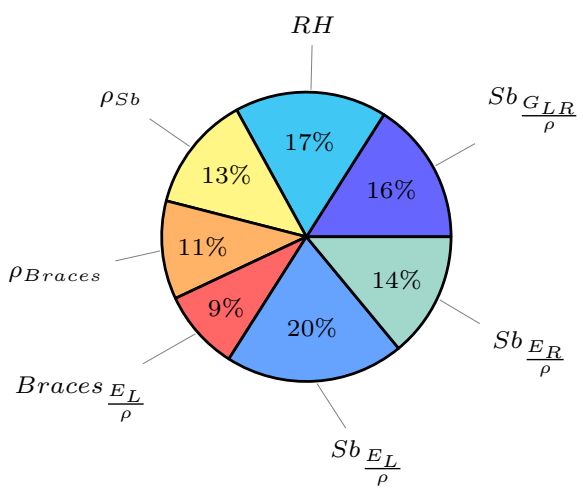

(b) Eigenfrequencies

(a)

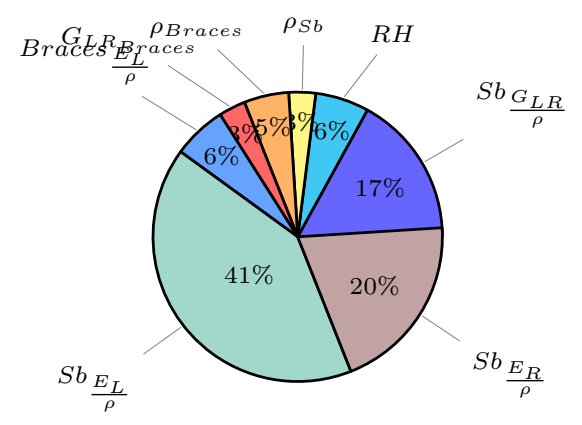

(a) Eigenvectors 